\title{
FINITE AUTOMORPHISM GROUPS OF LAMINATED NEAR-RINGS
}

\author{
by K. D. MAGILL, JR., P. R. MISRA and U. B. TEWARI \\ (Received 31st July, 1981)
}

\section{Introduction}

In [3] we initiated our study of the automorphism groups of a certain class of nearrings. Specifically, let $P$ be any complex polynomial and let $\mathscr{N}_{P}$ denote the near-ring of all continuous selfmaps of the complex plane where addition of functions is pointwise and the product $f g$ of two functions $f$ and $g$ in $\mathcal{N}_{P}$ is defined by $f g=f \circ P \circ g$. The nearring $\mathscr{N}_{P}$ is referred to as a laminated near-ring with laminating element $P$. In [3], we characterised those polynomials $P(z)=a_{n} z^{n}+a_{n-1} z^{n-1}+\ldots+a_{0}$ for which Aut $\mathscr{N}_{P}$ is a finite group. We are able to show that Aut $\mathscr{N}_{P}$ is finite if and only if $\operatorname{Deg} P \geqq 3$ and $a_{i} \neq 0$ for some $i \neq 0, n$. In addition, we were able to completely determine those infinite groups which occur as automorphism groups of the near-rings $\mathcal{N}_{\boldsymbol{P}}$. There are exactly three of them. One is GL(2) the full linear group of all real $2 \times 2$ nonsingular matrices and the other two are subgroups of GL(2). In this paper, we begin our study of the finite automorphism groups of the near-rings $\mathscr{N}_{\boldsymbol{p}}$. We get a result which, in contrast to the situation for the infinite automorphism groups, shows that infinitely many finite groups occur as automorphism groups of the near-rings under consideration. In addition to this and other results, we completely determine Aut $\mathscr{N}_{P}$ when the coefficients of $P$ are real and $\operatorname{Deg} P=3$ or 4 .

\section{Polynomials of arbitrary degree}

In this section we get some results without placing any restriction on $\operatorname{Deg} P$ other than it exceed two (the cases where $\operatorname{Deg} P=1$ and 2 were covered in [3]). We adhere to the notation of [3]. In particular, $\mathscr{C}$ denotes the complex plane regarded as a vector space over the real field and $\Pi(P)=\left\{P^{-1}(P(z)): z \in \mathscr{C}\right\}$. As in [3], the set $P^{-1}(P(0))$ will play a special role in our considerations and will be denoted by $Z(P)$. We will not hesitate to use without mention Corollary 2.3 of [3] which implies that for any complex polynomial $P$, Aut $\mathscr{N}_{P}$ is isomorphic to $L A(P)$ the group of all linear automorphisms $t$ of $\mathscr{C}$ with the property that $t[A] \in \Pi(P)$ for each $A \in \Pi(P)$. We begin our considerations with a sequence of lemmas.

Lemma 2.1. Let $t$ be a linear automorphism of $\mathscr{C}$ which has finite order and suppose $t(1)=1$. Then either $t$ is the identity or there exists a real number a such that

$$
t(x+y i)=x+a y-y i \text { for all } x+y i .
$$


Proof. There exist real numbers $a$ and $b$ such that $t(i)=a+b i$. One readily shows that for any positive integer $n$, we have

$$
t^{n}(i)=a\left(1+b+b^{2}+\ldots+b^{n-1}\right)+b^{n} i
$$

Since $t$ has finite order, $t^{n}$ is the identity for some integer $n$ and for that integer, it follows from (2.1.2) that

$$
b^{n}=1
$$

and

$$
a\left(1+b+b^{2}+\ldots+b^{n-1}\right)=0
$$

Since $b$ is real, we must have $b=1$ or $b=-1$. If $b=1$, it follows from (2.1.4) that $a=0$ which implies that $t$ is the identity. If $b=-1$, we have $t(i)=a-i$ which implies $t(x+y i)=x$ $+a y-y i$ and the proof is complete.

Lemma 2.2. Let $t$ be a linear automorphism of $\mathscr{C}$ which has finite order and suppose $t(1)=-1$. Then either $t(z)=-z$ for all $z \in \mathscr{C}$ or there exists a real number a such that

$$
t(x+y i)=-x+a y+y i \text { for all } x+y i
$$

Proof. Again, we have $t(i)=a+b i$ and one verifies that

$$
t^{n}(i)=a\left(1-b+b^{2}-b^{3}+\ldots+b^{n-1}\right)+b^{n} i
$$

for an odd integer $n$ while

$$
t^{n}(i)=a\left(-1+b-b^{2}+\ldots+b^{n-1}\right)+b^{n} i
$$

when $n$ is even. Since $t$ has finite order, $t^{n}$ must be the identity for some positive integer $n$. Regardless of whether $n$ is odd or even, it follows from (2.2.2) and (2.2.3) that $b^{n}=1$ and hence we must have $b=1$ or $b=-1$. If $b=1$ then $t(i)=a+i$ which implies $t(x+y i)$ $=-x+a y+y i$. If $b=-1$, then $n$ is even and it follows from (2.2.3) that $a=0$. In this instance $t(i)=-i$ and we have $t(z)=-z$ for all $z$.

The proofs of the next two lemmas are very similar to the proofs of Lemmas 2.1 and 2.2 and for that reason will be omitted.

Lemma 2.3. Let $t$ be a linear automorphism of $\mathscr{C}$ which has finite order and suppose $t(i)=i$. Then either $t$ is the identity or there exists a real number a such that

$$
t(x+y i)=-x+(a x+y) i \text { for all } x+y i .
$$

Lemma 2.4. Let $t$ be a linear automorphism of $\mathbb{C}$ which has finite order and suppose $t(i)=-i$. Then either $t(z)=-z$ for all $z \in \mathscr{C}$ or there exists a real number a such that 


$$
t(x+y i)=x+(a x-y) i \text { for all } x+y i .
$$

The next corollary is an immediate consequence of the previous four.

Corollary 2.5. Let $t$ be a linear automorphism of $\mathscr{C}$ which has finite order and suppose that either $t(1)=1, t(1)=-1, t(i)=i$ or $t(i)=-i$. Then the order of $t$ is either one or $t$ wo.

Definition 2.6. Let $\Gamma$ denote the linear automorphism of $\mathscr{C}$ which is defined by $\Gamma(z)$ $=\bar{z}$ for all $z \in \mathscr{C}$.

Corollary 2.7. Let $G$ be a finite subgroup of $G L(2)$ which contains $\Gamma$ and let $t$ be any element in $G$. Then all of the following statements are valid.

$$
\begin{aligned}
& \text { If } t(1)=1, \text { then either } t \text { is the identity or } t=\Gamma . \\
& \text { If } t(1)=-1, \text { then either } t(z)=-z \text { for all } z \text { or } t(z)=-\bar{z} \text { for all } z \text {. } \\
& \text { If } t(i)=i \text {, then either } t \text { is the identity or } t(z)=-\bar{z} \text { for all } z \text {. } \\
& \text { If } t(i)=-i \text {, then either } t(z)=-z \text { for all } z \text { or } t=\Gamma .
\end{aligned}
$$

Proof. We discuss only (2.7.1) as the remaining cases follow in the same manner. By Lemma 2.1, either $t$ is the identity or $t(x+y i)=x+a y-y i$ for some real number $a$. Suppose $a \neq 0$. Then $\Gamma \circ t$ is an element of $G$ which has infinite order. This, of course, is a contradiction so $a=0$ and $t=\Gamma$.

Corollary 2.8. Let $G$ be a finite subgroup of $G L(2)$ which contains $\Gamma$ and let $t$ be any element of $G$ which either maps a nonzero real number to a real number or a nonzero pure imaginary number to a pure imaginary number. Then $t$ satisfies one of the following conditions.

$$
\begin{gathered}
t \text { is the identity. } \\
\mathrm{t}=\Gamma . \\
t(z)=-z \text { for all } z \in \mathscr{C} . \\
t(z)=-\bar{z} \text { for all } z \in \mathscr{C} .
\end{gathered}
$$

Proof. Suppose $t(a)=b$ where $a \neq 0$. Then $t(1)=b / a$ and for any positive integer $n$, we have $t^{n}(1)=(b / a)^{n}$. Since $t$ has finite order, this implies $(b / a)^{n}=1$ for some $n$ which, in turn, implies $b / a=1$ or $b / a=-1$. It then follows from (2.7.1) and (2.7.2) that either (2.8.1), (2.8.2), (2.8.3) or (2.8.4) holds. The case where $t(a i)=b i$ follows in a similar manner.

The next result is an immediate consequence of the previous one. 
Corollary 2.9. Let $G$ be a finite subgroup of $G L(2)$ which contains $\Gamma$ and suppose each element of $G$ either maps a nonzero real number to a real number or a nonzero pure imaginary number to a pure imaginary number. Then $G$ is either isomorphic to $\mathbb{Z}_{2}$, the cyclic group of order two or to $\mathbb{K}_{4}$ the Klein four group.

Lemma 2.10. Suppose all the coefficients of $P$ are real. Then $\Gamma \in L A(P)$.

Proof. For any $z \in \mathscr{C}$, we have $P(\bar{z})=\overline{P(z)}$ so that $P\left(z_{1}\right)=P\left(z_{2}\right)$ if and only if $P\left(\Gamma\left(z_{1}\right)\right)$ $=P\left(\Gamma\left(z_{2}\right)\right)$. Lemma 3.1 of $[3]$ now applies.

We are now ready to state the first theorem of this section. Its proof is accomplished by simply piecing together various previous results.

Theorem 2.11. Let $P(z)=a_{n} z^{n}+a_{n-1} z^{n-1}+\ldots+a_{0}$ be a polynomial with real coefficients such that $n=\operatorname{Deg} P>3$ and $a_{i} \neq 0$ for some $i \neq 0$ or $n$. Suppose also that all the zeros of $P(z)-a_{0}$ are real. Then Aut $\mathcal{N}_{P}$ is isomorphic to either $\mathbb{Z}_{2}$ or $\mathbb{K}_{4}$.

Proof. By Theorem 3.6 of $[3], L A(P)$ is finite and $\Gamma \in L A(P)$ by Lemma 2.10 . By hypothesis, $Z(P)$ consists of real numbers and contains at least one nonzero real number. Since each $t \in L A(P)$ must map $Z(P)$ onto $Z(P)$ it follows from Corollary 2.9 that $L A(P)$ is isomorphic to either $\mathbb{Z}_{2}$ or $\mathbb{K}_{4}$.

We will later see that both situations occur. That is, there are polynomials $P$ for which Aut $\mathscr{N}_{P}$ is isomorphic to $\mathbb{Z}_{2}$ and others for which Aut $\mathscr{N}_{P}$ is isomorphic to $\mathbb{K}_{4}$.

Lemma 2.12. Let $P(z)=a_{n} z^{n}+a_{n-1} z^{n-1}+\ldots+a_{0} \quad\left(a_{n} \neq 0\right)$ and suppose $P^{-1}\left(P\left(z_{1}\right)\right)$ $=\left\{z_{1}, z_{2}, \ldots, z_{n}\right\}$ where the $z_{i}$ are all distinct. Then $z_{1}+z_{2}+\ldots+z_{n}=-\left(a_{n-1} / a_{n}\right)$.

Proof. $\left\{z_{1}, z_{2}, \ldots, z_{n}\right\}$ is the collection of zeros of the polynomial

$$
P(z)-P\left(z_{1}\right)=a_{n} z^{n}+a_{n-1} z^{n-1}+\ldots+a_{1} z+\left(a_{0}-P\left(z_{1}\right)\right)
$$

and it is well known that the sum of the zeros is $-\left(a_{n-1} / a_{n}\right)$.

Theorem 2.13. Let $P(z)=a_{n} z^{n}+a_{n-1} z^{n-1}+\ldots+a_{0}$ where $n=\operatorname{Deg} P \geqq 3$, all $a_{i}$ are real and $a_{n-1} \neq 0$. Then Aut $\mathscr{N}_{P}$ is isomorphic to $\mathbb{Z}_{2}$.

Proof. $L A(P)$ contains both the identity map and $\Gamma$ because of Lemma 2.10 . We need only show that there are no other elements in $L A(P)$. With this in mind, suppose $t \in L A(P)$ and choose $z_{1}$ such that

$$
P^{-1}\left(P\left(z_{1}\right)\right)=\left\{z_{1}, z_{2}, \ldots, z_{n}\right\}
$$

consists of $n$ distinct elements. Then $t\left[P^{-1}\left(P\left(z_{1}\right)\right)\right]=P^{-1}(P(w))$ for some $w$ and we have

$$
P^{-1}(P(w))=\left\{t\left(z_{1}\right), t\left(z_{2}\right), \ldots, t\left(z_{n}\right)\right\}
$$


From Lemma 2.12 we get

$$
t\left(a_{n-1} / a_{n}\right)=t\left(-\left(z_{1}+z_{2}+\ldots+z_{n}\right)\right)=-\left(t\left(z_{1}\right)+t\left(z_{2}\right)+\ldots+t\left(z_{n}\right)\right)=a_{n-1} / a_{n}
$$

which readily implies that $t(1)=1$. Now $L A(P)$ is finite by Theorem 3.6 of [3] so Corollary 2.7 now applies and we conclude that either $t$ is the identity or $t=\Gamma$.

In order to state our next theorem, we need to introduce a class of finite groups. Specifically, for each positive integer $n$, we denote by $G R_{n}$ the group of all $2 \times 2$ real matrices of the form

$$
\left[\begin{array}{rr}
a, & -b \\
b, & a
\end{array}\right] \text { and }\left[\begin{array}{rr}
a, & b \\
b, & -a
\end{array}\right]
$$

where $a=\cos (2 k \pi / n)$ and $b=\sin (2 k \pi / n) k=1,2,3, \ldots, n$. These are precisely the matrices which represent the linear automorphisms $t$ and $\bar{t}$ defined by $t(z)=\omega z$ and $\bar{t}(z)=\omega \bar{z}$ where $\omega$ is an $n^{\text {th }}$ root of unity. We will not hesitate to identify $G R_{n}$ with its corresponding group of linear automorphisms when it is convenient to do so. It is easy to see that $G R_{n}$ is a group of order $2 n$. It is commutative only when $n=1$ or 2 and in these cases it is isomorphic respectively to $\mathbb{Z}_{2}$ and $\mathbb{K}_{4}$. Since $G R_{3}$ contains six elements and is not commutative, it must necessarily be isomorphic to $S_{3}$ the symmetric group on three elements. And now we are in a position to state and prove

Theorem 2.14. Let $P(z)=a z^{n}+b z^{m}+c$ where $n \geqq 3, n>m>1$ and $a, b$ and $c$ are all real numbers with $a \neq 0 \neq b$. Then Aut $\mathscr{N}_{P}$ is isomorphic to $G R_{n-m}$.

Proof. According to Lemma 3.2 of [3] it is sufficient to show that Aut $\mathscr{N}_{P}$ is isomorphic to $G R_{n-m}$ where $Q(z)=z^{n}+d z^{m}$ and $d$ is a nonzero real number. Let $n-m$ $=k$ and we have

$$
Q(z)=z^{m}\left(z^{k}+d\right)
$$

Lemma 2.10 tells us that $\Gamma \in L A(Q)$. This fact will be used at various times throughout the remainder of the proof without explicit mention. In the first case we consider, $\Gamma$ turns out to be the only element in $L A(Q)$ other than the identity.

Case 1: $k=1$. Then $Q(z)=z^{n}+d z^{n-1}$ and it follows immediately from Theorem 2.13 that Aut $\mathscr{N}_{Q}$ is isomorphic to $\mathbb{Z}_{2}=G R_{1}$.

Case 2: $\quad k=2$. Here, we have $Z(Q)=\left\{0,(-d)^{\frac{1}{2}},-(-d)^{\frac{1}{2}}\right\}$ where $(-d)^{\frac{1}{2}}$ and $-(-d)^{\frac{1}{2}}$ are either both real numbers or both pure imaginary numbers depending upon whether or not $d$ is negative or positive. Thus, any element $t \in L A(Q)$ must either carry a nonzero real number to a nonzero real number or a pure imaginary number to a pure imaginary number. It follows from Corollary 2.9 that $L A(Q)$ is isomorphic to either $\mathbb{Z}_{2}$ or $\mathbb{K}_{4}$. The latter is, in fact, the case and to see that, all we need to do is exhibit a $t \in L A(Q)$ which is distinct from the identity and from $\Gamma$. Consider $t(z)=-z$. Then $Q(t(z))$ is either $Q(z)$ 
or $-Q(z)$ depending upon whether $m$ is even or odd. In either event we have $Q\left(z_{1}\right)$ $=Q\left(z_{2}\right)$ if and only if $Q\left(t\left(z_{1}\right)\right)=Q\left(t\left(z_{2}\right)\right)$ and it follows from Lemma 3.1 of [3] that $t \in L A(Q)$. Thus $L A(Q)$ is isomorphic to $\mathbb{K}_{4}=G R_{2}$.

Case 3: $k \geqq 3$ and $k \neq 4$. Let $\left\{\omega_{1}, \omega_{2}, \ldots, \omega_{k}\right\}$ be the $k^{\text {th }}$ roots of unity and for each $i$ $=1,2,3, \ldots, k$ define

$$
t_{i}(z)=\omega_{i} z \text { for all } z \in \mathscr{C}
$$

and

$$
\bar{t}_{i}(z)=\omega_{i} \bar{z} \text { for all } z \in \mathscr{C}
$$

Then we have

$$
G R_{k}=\left\{t_{i}\right\}_{i=1}^{k} \cup\left\{\bar{t}_{i}\right\}_{i=1}^{k}
$$

One readily shows that

$$
Q\left(t_{i}(z)\right)=\omega_{i}^{m} Q(z)
$$

and

$$
Q\left(\vec{t}_{i}(z)\right)=\omega_{i}^{m} \overline{P(z)}
$$

from whence it readily follows that $Q\left(z_{1}\right)=Q\left(z_{2}\right)$ if and only if $Q\left(t_{i}\left(z_{1}\right)\right)=Q\left(t_{i}\left(z_{2}\right)\right)$ if and only if $Q\left(\bar{t}_{i}\left(z_{1}\right)\right)=Q\left(\bar{t}_{i}\left(z_{2}\right)\right)$. Thus

$$
G R_{k} \subset L A(Q)
$$

by Lemma 3.1 of [3]. We will show, in fact, that $G R_{k}=L A(Q)$. Let $r=|d|^{1 / k}$ and let $\left\{\alpha_{1}, \alpha_{2}, \ldots, \alpha_{k}\right\}$ denote the $k^{\text {th }}$ roots of -1 if $d>0$ and the $k^{\text {th }}$ roots of 1 if $d<0$. Then

$$
Z(Q)=\left\{0, r \alpha_{1}, r \alpha_{2}, \ldots, r \alpha_{k}\right\}
$$

Now we take any element $t \in L A(Q)$ and since $t$ maps $Z(Q)$ bijectively onto $Z(Q)$ the hypothesis of Lemma 4.1 of [3] is satisfied (note: it is not satisfied when $k=4$ ). It follows that either $t(z)=w z$ or $t(z)=w \bar{z}$ for an appropriate complex number $w \neq 0$. Suppose the former holds. Then $w \alpha_{1}=t\left(\alpha_{1}\right)=\alpha_{i}$ for some $i$ which implies $w=\alpha_{i} / \alpha_{1}$. It follows that $w$ is a $k^{\text {th }}$ root of unity regardless of whether the $\alpha_{j}$ are $k^{\text {th }}$ roots of -1 or $k^{\text {th }}$ roots of 1 . Thus, $t=t_{j}$ for some $j$ which means $t \in G R_{k}$. Similarly, one shows that if $t(z)=w \bar{z}$, then $t=\bar{t}_{j}$ for some $j$ and hence, in this case also, $t \in G R_{k}$. Consequently, $G R_{k}$ $=L A(Q)$ and we conclude that Aut $\mathscr{N}_{Q}$ is isomorphic to $G R_{k}$ when $k \geqq 3$ and $k \neq 4$. It remains for us to treat

Case 4: $k=4$. With one exception, this case is identical to the preceding case even 
up to the point where we have $G R_{4} \subset L A(Q)$. The exception occurs because we cannot use Lemma 4.1 of [3] to show $L A(Q) \subset G R_{4}$. Instead, we have to do this directly. We will discuss the details only in the case $d>0$ which means that $\left\{\alpha_{1}, \alpha_{2}, \alpha_{3}, \alpha_{4}\right\}$ represent the $4^{\text {th }}$ roots of -1 . Let $q=(r \sqrt{2} / 2), v=1+i$ and $w=-i+1$ and we have

$$
Z(Q)=\{0, q v,-q v, q w,-q w\}
$$

Since any $t \in L A(Q)$ must map $Z(Q)$ bijectively onto itself, $t(v)$ can be any one of the vectors $v,-v, w,-w$ and $t(w)$ can be any one of the remaining two vectors which are each linearly independent from $t(v)$. With some calculation one shows that $t$ must be given by one of the following equations:

$$
\begin{aligned}
& t(z)=z, \quad t(z)=-z, \quad t(z)=i z, \quad t(z)=-i z \\
& t(z)=\bar{z}, \quad t(z)=-\bar{z}, \quad t(z)=i \bar{z}, \quad t(z)=-i \bar{z} .
\end{aligned}
$$

In other words, $t \in G R_{4}$. We have thus shown that $G R_{4}=L A(Q)$ and the proof is now complete.

\section{Third and fourth degree polynomials}

Theorem 4.3 of [3] tells us that if $\operatorname{Deg} P=1$ or $\operatorname{Deg} P=2$ and the coefficient of $z$ is zero then Aut $\mathcal{N}_{p}$ is isomorphic to $G L(2)$. It further tells us that if $\operatorname{Deg} P=2$ and the coefficient of $z$ is not zero then Aut $\mathcal{N}_{p}$ is isomorphic to $G_{1}$, the group of all real $2 \times 2$ matrices of the form

$$
\left[\begin{array}{ll}
1, & a \\
0, & b
\end{array}\right] \text { where } b \neq 0
$$

We have therefore completely determined Aut $\mathcal{N}_{p}$ when $\operatorname{Deg} P$ is either one or two. In this section we supplement this information by determining Aut $\mathscr{N}_{p}$ when $P$ has real coefficients and $\operatorname{Deg} P=3$ or 4 . The result for $\operatorname{Deg} P=3$ is an immediate consequence of several of our preceeding results.

Theorem 3.1. Let $P(z)=a z^{3}+b z^{2}+c z+d$ be a cubic polynomial with real coefficients. Then

$$
\begin{aligned}
& \text { Aut } \mathscr{N}_{P} \text { is isomorphic to } \mathbb{Z}_{2} \text { if } b \neq 0, \\
& \text { Aut } \mathscr{N}_{P} \text { is isomorphic to } \mathbb{K}_{4} \text { if } b=0 \text { and } c \neq 0 \text {, } \\
& \text { Aut } \mathscr{N}_{P} \text { is isomorphic to } G_{c} \text { if } b=0=c .
\end{aligned}
$$

Proof. (3.1.1) follows from Theorem 2.13, (3.1.2) follows from Theorem 2.14 and (3.1.3) follows from Theorem 4.4 of [3]. 
Theorem 3.2. Let $P(z)=a_{4} z^{4}+a_{3} z^{3}+a_{2} z^{2}+a_{1} z+a_{0}$ be a fourth degree polynomial with real coefficients. Then we have the following:

Aut $\mathscr{N}_{P} \quad$ is isomorphic to $\mathbb{Z}_{2}$ if $a_{3} \neq 0$.

Aut $\mathscr{N}_{P}$ is isomorphic to $\mathbb{Z}_{2}$ if $a_{3}=0, a_{2} \neq 0$ and $a_{1} \neq 0$.

Aut $\mathscr{N}_{P}$ is isomorphic to $\mathbb{K}_{4}$ if $a_{3}=0, a_{2} \neq 0$ and $a_{1}=0$.

Aut $\mathcal{N}_{P} \quad$ is isomorphic to $S_{3}$ if $a_{3}=0, \quad a_{2}=0$ and $a_{1} \neq 0$.

Aut $\mathscr{N}_{P}$ is isomorphic to $G_{c}$ if $a_{3}=0, a_{2}=0$ and $a_{1}=0$.

Proof. (3.2.1) follows from Theorem 2.13, both (3.2.3) and (3.2.4) follow from Theorem 2.14 and (3.2.5) follows from Theorem 4.3 of [3]. It remains for us to verify (3.2.2). We need only show that $L A(Q)$ is isomorphic to $\mathbb{Z}_{2}$ where $Q(z)=z^{4}+a z^{2}+b z$ and $a \neq 0 \neq b$.

Case 1: $Z(Q)$ consists entirely of real numbers.

Then $Z(Q)$ is either $\{0, r\},\left\{0, r_{1}, r_{2}\right\}$ or $\left\{0, r_{1}, r_{2}, r_{3}\right\}$. Let $t \in L A(Q)$. Then $t(1)=d$ and $d$ must be real since otherwise $t$ would not map $Z(Q)$ into $Z(Q)$. Since $t$ has finite order $n$, we have $1=t^{n}(1)=d^{n}$ which implies $t(1)=1$ or $t(1)=-1$. It follows that either $t$ is the identity on real numbers or $t$ takes every real number to its negative. If $Z(Q)$ is either $\{0, r\}$ or $\left\{0, r_{1}, r_{2}, r_{3}\right\}$ it evidently contains some real number and not its negative so that in these instances we must have $t(1)=1$. If $Z(Q)=\left\{0, r_{1}, r_{2}\right\}$, we may assume $Q(z)=$ $z\left(z-r_{1}\right)^{2}\left(z-r_{2}\right)$ which implies $r_{2}=-2 r_{1}$ (since the coefficient of $z^{3}$ is zero). Since $r_{2} \neq-r_{1}$ we must again have $t(1)=1$. It now follows from Corollary 2.7 that $t$ must be either the identity or $\Gamma$.

Case 2: $Z(Q)$ contains nonreal numbers.

Since $0 \in Z(Q)$ and complex roots occur in conjugate pairs, $Z(Q)$ must contain exactly two complex numbers. Moreover, $Z(Q)$ must contain a nonzero real number since the coefficient of $z$ is not 0 while the constant term is. Thus, we have

$$
Z(Q)=\{0, r, v, \bar{v}\}
$$

where $r \neq 0$ is real and $v$ is not. This means that we have

$$
Q(z)=z(z-r)(z-v)(z-\bar{v})
$$

Now let $t \in L A(Q)$. We want to show that $t(r)=r$. Suppose, to the contrary, that $t(r) \neq r$. There is no loss in generality if we assume that $t(r)=v$. Let $v=c+d i$. Since the coefficient of $z^{3}$ is zero, we have $r+v+\bar{v}=0$ which implies $c=-r / 2$. Let $k=-2 d / r$ and we have

$$
v=-\frac{r}{2}(1+k i), \quad \bar{v}=-\frac{r}{2}(1-k i)
$$


Now $t(r)=v$ implies $t(1)=v / r$ and from (5.16.8) we get

$$
t(1)=-\frac{1}{2}(1+k i)
$$

Next choose a real number $r_{1}$ between 0 and $r$ such that $P^{-1}\left(P\left(r_{1}\right)\right)$ contains another real number $r_{2}$ distinct from $r_{1}$ which also lies between 0 and $r$. Since $t \in L A(Q)$, we have $Q\left(t\left(r_{1}\right)\right)=Q\left(t\left(r_{2}\right)\right)$. From (3.2.9) we see that

$$
t\left(r_{j}\right)=-\frac{r_{j}}{2}(1+k i) \quad j=1,2
$$

Next, use (3.2.7), (3.2.8) and (3.2.9) to compute each $Q\left(t\left(r_{j}\right)\right)$. Setting $Q\left(t\left(r_{1}\right)\right)=Q\left(t\left(r_{2}\right)\right)$ and equating imaginary parts we obtain

$$
\left(1+k^{2}\right) / 4=\left[r^{2}\left(r_{1}^{2}-r_{2}^{2}\right)-\left(r_{1}^{4}-r_{2}^{4}\right)\right] /\left[r^{2}\left(r_{1}^{2}-r_{2}^{2}\right)-2\left(r_{1}^{4}-r_{2}^{4}\right)+r^{3}\left(r_{1}-r_{2}\right)\right]
$$

By setting $Q\left(r_{1}\right)=Q\left(r_{2}\right)$ we obtain

$$
\left(1+k^{2}\right) / 4=\left[r^{2}\left(r_{1}^{2}-r_{2}^{2}\right)-\left(r_{1}^{4}-r_{2}^{4}\right)\right] /\left[r^{2}\left(r_{1}^{2}-r_{2}^{2}\right)-r^{3}\left(r_{1}-r_{2}\right)\right] .
$$

From (3.2.11) and (3.2.12) we obtain $r_{1}^{4}-r_{2}^{4}=r^{3}\left(r_{1}-r_{2}\right)$ and by replacing $r_{1}^{4}-r_{2}^{4}$ by $r^{3}\left(r_{1}-r_{2}\right)$ in either (3.2.11) or (3.2.12) we get $\left(1+k^{2}\right) / 4=1$ or, equivalently,

$$
k^{2}=3
$$

From (3.2.7) and (3.2.8), one shows that the coefficient of $z^{2}$ is $|v|^{2}-r^{2}$. But (3.2.13) and (3.2.8) together imply $|v|^{2}-r^{2}=0$. This is the contradiction we seek for we are considering the case $Q(z)=z^{4}+a z^{2}+b z$ where neither $a$ nor $b$ are zero. Therefore we must indeed have $t(r)=r$ and it follows from Corollary 3.7 that either $t$ is the identity or $t=\Gamma$. Thus, $L A(Q)$ is isomorphic to $\mathbb{Z}_{2}$ and the proof is complete.

Some concluding remarks are in order. It is evident that much remains to be done in order to completely determine Aut $\mathscr{N}_{p}$ for an arbitrary complex polynomial $P$. The next step is probably to determine Aut $\mathcal{N}_{p}$ when $\operatorname{Deg} P=5$ and $P$ has real coefficients. Although many of the special cases follow from previous results and techniques used in this paper and in [3], we are still unable to completely solve the problem for $5^{\text {th }}$ degree polynomials. In particular, we are unable to determine $L A(P)$ whenever

$$
Z(P)=\{0, v, \bar{v},-v,-\bar{v}\}
$$

Once Aut $\mathscr{N}_{p}$ is known for $\operatorname{Deg} P=5$, we might have enough information to make some educated guesses at what the general results (if such exist) might be.

Acknowledgement. This project was begun while the second author was a faculty member of Universidade Federal de Goias and the first and third authors were visiting there. The authors wish to thank their friends and colleagues at the University for their kind hospitality and both the University and $\mathrm{CNPq}$ for their generous financial support. 


\section{REFERENCES}

1. K. D. Magill, Jr., Semigroups and near-rings of continuous functions, General Topology and its Relations to Modern Analysis and Algebra, III, Proc. Third Prague Top. Symp. 1971 (Academia, 1972), 283-288.

2. K. D. Magill, Jr., Automorphism groups of laminated near-rings, Proc. Edinburgh Math. Soc. 23 (1980), 97-102.

3. K. D. Magill, Jr., P. R. Misra and U. B. Tewari, Automorphism groups of laminated nearrings determined by complex polynomials, Proc. Edinburgh Math. Soc. 26 (1983), 73-84.

State University of New York at Buffalo

Universidade Estadual, de CAMPINAS IIT Kanpur India 